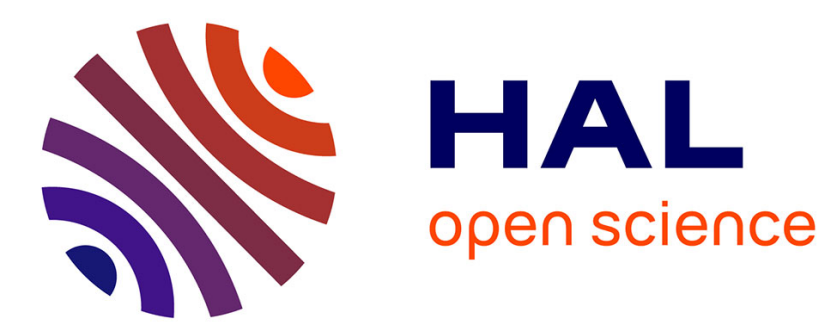

\title{
Color Object Recognition Based On Clifford Fourier Transform
}

\author{
José Mennesson, Christophe Saint-Jean, Laurent Mascarilla
}

\section{To cite this version:}

José Mennesson, Christophe Saint-Jean, Laurent Mascarilla. Color Object Recognition Based On Clifford Fourier Transform. Leo Dorst and Joan Lasenby. Guide to Geometric Algebra in Practice, Springer London, pp.175-191, 2011, 978-0-85729-810-2. 10.1007/978-0-85729-811-9_9 . hal00808093

\section{HAL Id: hal-00808093 https://hal.science/hal-00808093}

Submitted on 4 Apr 2013

HAL is a multi-disciplinary open access archive for the deposit and dissemination of scientific research documents, whether they are published or not. The documents may come from teaching and research institutions in France or abroad, or from public or private research centers.
L'archive ouverte pluridisciplinaire HAL, est destinée au dépôt et à la diffusion de documents scientifiques de niveau recherche, publiés ou non, émanant des établissements d'enseignement et de recherche français ou étrangers, des laboratoires publics ou privés. 


\title{
Color Object Recognition Based On Clifford Fourier Transform
}

\author{
Jose Mennesson, Christophe Saint-Jean and Laurent Mascarilla
}

\begin{abstract}
The aim of this paper is to propose two different approaches for color object recognition, both using the recently defined color Clifford Fourier transform. The first one deals with so-called Generalized Fourier Descriptors, the definition of which relies on plane motion group actions. The proposed color extension leads to more compact descriptors, with lower complexity and better recognition rates, than the already existing descriptors based on the processing of the $\mathrm{r}, \mathrm{g}$ and $\mathrm{b}$ channels separately (later referred as marginal processing). The second approach concerns color phase correlation for color images. The idea here is to generalize in the Clifford framework the usual means of measuring correlation from the well-known shift theorem. Both methods necessitate to choose a bivector $B$ of $\mathbb{R}_{4,0}$ which corresponds to an analysis plane in the color space. The relevance of proposed methods for classification purposes is discussed on several color image database. In particular, the influence of parameter $B$ is studied regarding the type of images.
\end{abstract}

\section{Introduction}

Most of already existing works on image recognition make use of discriminative and invariant descriptors. Among them, moment-based descriptors [7] such as $\mathrm{Hu}$ invariants, Legendre moments or Zernike moments are well known. Beside these approaches, SIFT (Scale-Invariant Feature Transform) descriptors are a popular choice giving very good results [11]. An alternative to these methods is to define descriptors in the frequency domain. In this framework, our paper concerns two extensions

Jose Mennesson

Lab. MIA, La Rochelle University, France, e-mail: jose.mennesson@ univ-lr.fr

Christophe Saint-Jean

Lab. MIA, La Rochelle University, France, e-mail: christophe.saint-jean@univ-lr.fr

Laurent Mascarilla

Lab. MIA, La Rochelle University, France, e-mail: laurent.mascarilla@univ-lr.fr 
of existing methods based on a Fourier transform. Clearly, Fourier coefficients do not respect the classical invariances (translation, rotation and scale) and must be processed to obtain invariant descriptors. This paper proposes an extension of a recent advance concerning invariant Generalized Fourier Descriptors $(G F D)$ defined by F. Smach et al. [15]. The extension of these descriptors to color images is generally based on a marginal processing of the three channels (r,g,b). Then, descriptors extracted from each channel are concatenated to form the description vector. In order to avoid this marginal processing, our proposal is to extract descriptors from a color Clifford Fourier transform as defined by Batard et al. [1]. A second proposal is the extension of the classical color phase correlation by means of the same Fourier transform.

\section{A Clifford Fourier transform for color image processing}

As relating to color image processing, the usual Fourier transform corresponds in fact to 3 two-dimensional Fourier transforms applied on each color channel, that is a marginal processing. To emphasize the role of color, several authors have proposed to embed the color space in a more pertinent and meaningful geometric space such as the space of quaternions. For instance, Ell and Sangwine [6] propose a luminance/chrominance Fourier analysis replacing the imaginary complex $i$ by the quaternion $\mu=\frac{i+j+k}{\sqrt{3}}$ corresponding to the gray-level axis. It already appears in this work that one has to focus on an analysis direction (here given by $\mu$ ).

Recently, Batard et al. [1] have defined a Fourier transform for $L^{2}\left(\mathbb{R}^{m} ; \mathbb{R}^{n}\right)$ functions. This one is mathematically rigorous and clarifies relations between the Fourier transform and the action of the translation group through an action of a spinor group. They show that the previously proposed generalizations for color images (i.e. $n=3$, the number of color channels) are particular cases of their definition. In this paper, only the particular case $m=2$ and $n=3$ is considered and described briefly in the following. Firstly, in the equation of the classical 2D Fourier transform,

$$
\hat{f}\left(u_{1}, u_{2}\right)=\int_{\mathbb{R}^{2}} f\left(x_{1}, x_{2}\right) \mathrm{e}^{-\mathrm{i}\left(u_{1} x_{1}+u_{2} x_{2}\right)} \mathrm{d} x_{1} \mathrm{~d} x_{2}
$$

the term $\mathrm{e}^{-\mathrm{i}\left(u_{1} x_{1}+u_{2} x_{2}\right)}\left(=\mathrm{e}^{-\mathrm{i}\langle\mathbf{u}, \mathbf{x}\rangle}\right.$ with $\mathbf{u}=\left(u_{1}, u_{2}\right)$ and $\left.\mathbf{x}=\left(x_{1}, x_{2}\right)\right)$ rotates $f\left(x_{1}, x_{2}\right)$ in the complex plane $\mathbb{C}$. From a mathematical point of view, it corresponds to the action of the group $S^{1}$ on $\mathbb{C}$ which can be identified as the group action of $S O(2)$ on $\mathbb{R}^{2}$. In order to generalize this principle to color images, one have to consider the action of the matrix group $S O(3)$ on $\mathbb{R}^{3}$. As described in [1], a general and elegant expression may be written if the function corresponding to the color image is embedded in the Clifford algebra $\mathbb{R}_{4,0}$ :

$$
f(x, y)=r(x, y) \mathbf{e}_{\mathbf{1}}+g(x, y) \mathbf{e}_{\mathbf{2}}+b(x, y) \mathbf{e}_{\mathbf{3}}+0 \mathbf{e}_{\mathbf{4}}
$$


Within this framework, the rotation of a vector $\mathbf{v}$ by the angle $-\theta$, in the plane generated by a unitary bivector $B$ is given by the action $s \mathbf{v} s^{-1}$ of a spinor $s$ and can be written as

$$
\mathbf{v} \rightarrow s^{-1} \mathbf{v} s=\mathrm{e}^{\frac{\theta}{2} B} \mathbf{v} \mathrm{e}^{-\frac{\theta}{2} B}
$$

For this type of functions, the following Fourier transform is considered :

$$
\widehat{f}_{B}(\mathbf{u})=\int_{\mathbb{R}^{2}} \mathrm{e}^{\frac{\langle\mathbf{u}, \mathbf{x}\rangle}{2} I_{4} B} \mathrm{e}^{\frac{\langle\mathbf{u}, \mathbf{x}\rangle}{2} B} f(\mathbf{x}) \mathrm{e}^{-\frac{\langle\mathbf{u}, \mathbf{x}\rangle}{2} B} \mathrm{e}^{-\frac{\langle\mathbf{u}, \mathbf{x}\rangle}{2} I_{4} B} \mathrm{~d} \mathbf{x}
$$

where $I_{4}$ is the pseudo-scalar of $\mathbb{R}_{4,0}, I_{4} B$ is an unitary bivector orthogonal to $B$, $\mathbf{u}=\left(u_{1}, u_{2}\right)$ and $\mathbf{x}=\left(x_{1}, x_{2}\right)$. From the geometric point of view, two independent rotations in orthogonal planes are acting on $f(\mathbf{x})$. As these rotations are of same angle, the chosen Fourier transform involves isoclinic rotations of $f$ in $\mathbb{R}^{4}[10]$. Let us emphasize that considering more general rotations in $\mathbb{R}^{4}$ leads to other definitions of the Fourier transform and yields additional parameters which are hard to set in practice.

\subsection{The shift theorem for the Clifford Fourier Transform}

The color phase correlation subsequently proposed relies on the Fourier Shift theorem which states that a translation in the spatial domain induces a phase shift in the frequency domain. By construction, this property still holds for our transform and takes the following form.

Theorem 1. Let $f, g \in L^{2}\left(\mathbb{R}^{2}, \mathbb{R}^{3}\right), B$ a unit bivector in $\mathbb{R}_{4,0}$ and $\boldsymbol{\Delta}=\left(\Delta_{1}, \Delta_{2}\right)$ the vector containing the translation parameters.

If $g(\mathbf{x})=f(\mathbf{x}+\boldsymbol{\Delta})$ then

$$
\widehat{g_{B}}(\mathbf{u})=\mathrm{e}^{-\frac{\langle\mathbf{u}, \boldsymbol{\Delta}\rangle}{2} I_{4} B} \mathrm{e}^{-\frac{\langle\mathbf{u}, \boldsymbol{\Delta}\rangle}{2} B} \widehat{f_{B}}(\mathbf{u}) \mathrm{e}^{\frac{\langle\mathbf{u}, \boldsymbol{\Delta}\rangle}{2} B} \mathrm{e}^{\frac{\langle\mathbf{u}, \boldsymbol{\Delta}\rangle}{2} I_{4} B}
$$

Proof. The proof is essentially based on the fact that rotations in orthogonal planes can commute. Let $\mathbf{x}_{\boldsymbol{\Delta}}=\mathbf{x}+\boldsymbol{\Delta}$.

$$
\begin{aligned}
\widehat{g_{B}}(\mathbf{u}) & =\int_{\mathbb{R}^{2}} \mathrm{e}^{\frac{\langle\mathbf{u}, \mathbf{x}\rangle}{2}\left(B+I_{4} B\right)} f(\mathbf{x}+\boldsymbol{\Delta}) \mathrm{e}^{-\frac{\langle\mathbf{u}, \mathbf{x}\rangle}{2}\left(B+I_{4} B\right)} \mathrm{d} \mathbf{x} \\
& =\int_{\mathbb{R}^{2}} \mathrm{e}^{\frac{\left\langle\mathbf{u},\left(\mathbf{x}_{\boldsymbol{\Delta}}-\boldsymbol{\Delta}\right)\right\rangle}{2}\left(B+I_{4} B\right)} f\left(\mathbf{x}_{\boldsymbol{\Delta}}\right) \mathrm{e}^{-\frac{\left\langle\mathbf{u},\left(\mathbf{x}_{\mathbf{\Delta}}-\boldsymbol{\Delta}\right)\right\rangle}{2}\left(B+I_{4} B\right)} \mathrm{d} \mathbf{x}_{\boldsymbol{\Delta}} \\
& =\int_{\mathbb{R}^{2}} \mathrm{e}^{-\frac{\langle\mathbf{u}, \boldsymbol{\Delta}\rangle}{2}\left(B+I_{4} B\right)} \mathrm{e}^{\frac{\langle\mathbf{u}, \mathbf{x} \boldsymbol{\Delta}\rangle}{2}\left(B+I_{4} B\right)} f\left(\mathbf{x}_{\boldsymbol{\Delta}}\right) \mathrm{e}^{-\frac{\left\langle\mathbf{u}, \mathbf{x}_{\boldsymbol{\Delta}}\right\rangle}{2}\left(B+I_{4} B\right)} \mathrm{e}^{\frac{\langle\mathbf{u}, \boldsymbol{\Delta}\rangle}{2}\left(B+I_{4} B\right)} \mathrm{d} \mathbf{x}_{\boldsymbol{\Delta}} \\
& =\mathrm{e}^{-\frac{\langle\mathbf{u}, \boldsymbol{\Delta}\rangle}{2}\left(B+I_{4} B\right)}\left(\int_{\mathbb{R}^{2}} \mathrm{e}^{\frac{\left\langle\mathbf{u}, \mathbf{x}_{\boldsymbol{\Delta}}\right\rangle}{2}\left(B+I_{4} B\right)} f\left(\mathbf{x}_{\boldsymbol{\Delta}}\right) \mathrm{e}^{-\frac{\left\langle\mathbf{u}, \mathbf{x}_{\boldsymbol{\Delta}}\right\rangle}{2}\left(B+I_{4} B\right)} \mathrm{d} \mathbf{x}_{\boldsymbol{\Delta}}\right) \mathrm{e}^{\frac{\langle\mathbf{u}, \boldsymbol{\Delta}\rangle}{2}\left(B+I_{4} B\right)} \\
& =\mathrm{e}^{-\frac{\langle\mathbf{u}, \boldsymbol{\Delta}\rangle}{2}\left(B+I_{4} B\right)} \widehat{f}_{B}(\mathbf{u}) \mathrm{e}^{\frac{\langle\mathbf{u}, \boldsymbol{\Delta}\rangle}{2}\left(B+I_{4} B\right)}
\end{aligned}
$$


Even if (4) is more compact than (3), this formulation emphasizes that two independent rotations apply. Simpler equations can be obtained using the decomposition of $f$ as the sum of its parallel projection $f_{\| B}$ on the plane defined from $B$ and its perpendicular projection $f_{\perp B}$ on the plane defined from $I_{4} B$ (see later for details on the decomposition). Skipping some technical details, (2) can be rewritten following this decomposition as

$$
\widehat{f_{B}}(\mathbf{u})=\widehat{f_{\| B}}(\mathbf{u})+\widehat{f_{\perp B}}(\mathbf{u})
$$

where

$$
\begin{gathered}
\widehat{f_{\| B}}(\mathbf{u})=\int_{\mathbb{R}^{2}} \mathrm{e}^{\frac{\langle\mathbf{u}, \mathbf{x}\rangle}{2} B} f_{\| B}(\mathbf{x}) \mathrm{e}^{-\frac{\langle\mathbf{u}, \mathbf{x}\rangle}{2} B} \mathrm{~d} \mathbf{x}=\int_{\mathbb{R}^{2}} f_{\| B}(\mathbf{x}) \mathrm{e}^{-\langle\mathbf{u}, \mathbf{x}\rangle B} \mathrm{~d} \mathbf{x} \\
\widehat{f_{\perp B}}(\mathbf{u})=\int_{\mathbb{R}^{2}} \mathrm{e}^{\frac{\langle\mathbf{u}, \mathbf{x}\rangle}{2} I_{4} B} f_{\perp B}(\mathbf{x}) \mathrm{e}^{-\frac{\langle\mathbf{u}, \mathbf{x}\rangle}{2} I_{4} B} \mathrm{~d} \mathbf{x}=\int_{\mathbb{R}^{2}} f_{\perp B}(\mathbf{x}) \mathrm{e}^{-\langle\mathbf{u}, \mathbf{x}\rangle I_{4} B} \mathrm{~d} \mathbf{x}
\end{gathered}
$$

Later on, (6) and (7) will provide a practical and efficient way to implement our transform. According the same decomposition, Theorem 1 becomes

Theorem 2. Let $f, g \in L^{2}\left(\mathbb{R}^{2}, \mathbb{R}^{3}\right)$ and $B$ a unit bivector in $\mathbb{R}_{4,0}$. If $g(\mathbf{x})=f(\mathbf{x}+\boldsymbol{\Delta})$ then

$$
\begin{gathered}
\widehat{g_{\| B}}(\mathbf{u})=\mathrm{e}^{-\frac{\langle\mathbf{u}, \boldsymbol{\Delta}\rangle}{2} B} \widehat{f_{\| B}}(\mathbf{u}) \mathrm{e}^{\frac{\langle\mathbf{u}, \boldsymbol{\Delta}\rangle}{2} B}=\widehat{f_{\| B}}(\mathbf{u}) \mathrm{e}^{\langle\mathbf{u}, \boldsymbol{\Delta}\rangle B} \\
\widehat{g_{\perp B}}(\mathbf{u})=\mathrm{e}^{-\frac{\langle\mathbf{u}, \boldsymbol{\Delta}\rangle}{2} I_{4} B} \widehat{f_{\perp B}}(\mathbf{u}) \mathrm{e}^{\frac{\langle\mathbf{u}, \boldsymbol{\Delta}\rangle}{2} I_{4} B}=\widehat{f_{\perp B}}(\mathbf{u}) \mathrm{e}^{\langle\mathbf{u}, \boldsymbol{\Delta}\rangle I_{4} B}
\end{gathered}
$$

A unit bivector $B$ can be obtained from the geometric product of two unit orthogonal vectors as $B=\mathbf{c} \wedge \mathbf{e}_{\mathbf{4}}$ or $B=\mathbf{c}_{\mathbf{1}} \wedge \mathbf{c}_{\mathbf{2}}$ (where $\mathbf{c}, \mathbf{c}_{\mathbf{1}}$ and $\mathbf{c}_{\mathbf{2}}$ are RGB colors). These two settings appear to be analogous up to a sign change since the dualization $I_{4} B$ of $B$ gives also a bivector of the other form. In the following, only the choice $B=\mathbf{c} \wedge \mathbf{e}_{\mathbf{4}}$ will be considered in the experiments.

\subsection{Computation of the Clifford Fourier Transform}

The Clifford Fourier Transform can be efficiently computed using two complex FFTs. Whereas $\left\{\mathbf{c}, \mathbf{e}_{\mathbf{4}}\right\}$ is a trivial basis for the plane given by $B$, an orthonormal basis $\{\mathbf{v}, \mathbf{w}\}$ for the plane generated by $I_{4} B$ has to be constructed. A possible solution is to choose a unit vector $\mu$ with no $\mathbf{e}_{\mathbf{4}}$ component and different from $\mathbf{c}^{1}$. Vector $\mathbf{v}$ is taken as the rejection of $\mu$ on $\mathbf{c}$ and $\mathbf{w}$ as an orthogonal vector to $\mathbf{v}$ in subspace represented by blade $I_{4} B$ (see Fig. 1). Then, the function $f$ can decomposed as

$$
\begin{aligned}
f(\mathbf{x}) & =\quad \begin{array}{c}
f_{\| B}(\mathbf{x}) \\
\end{array}=\mathbf{c}[(f(\mathbf{x}) \cdot \mathbf{c})+(f(\mathbf{x}) \cdot \mathbf{c} B) B]+\mathbf{v}\left[(f(\mathbf{x}) \cdot \mathbf{v})+\left(f(\mathbf{x}) \cdot \mathbf{v} I_{4} B\right) I_{4} B\right]
\end{aligned}
$$

\footnotetext{
${ }^{1}$ A typical setting for $\mu$ is $\left(\mathbf{e}_{\mathbf{1}}+\mathbf{e}_{\mathbf{2}}+\mathbf{e}_{\mathbf{3}}\right) / \sqrt{3}$ which corresponds to select the achromatic axis.
} 
Each of the two square brackets can identified as complex number since $B B=$ $I_{4} B I_{4} B=-1$. The computation of $\widehat{f}_{B}$ is now reduced to the computation of two usual Fourier transforms of a real function and of a complex function.

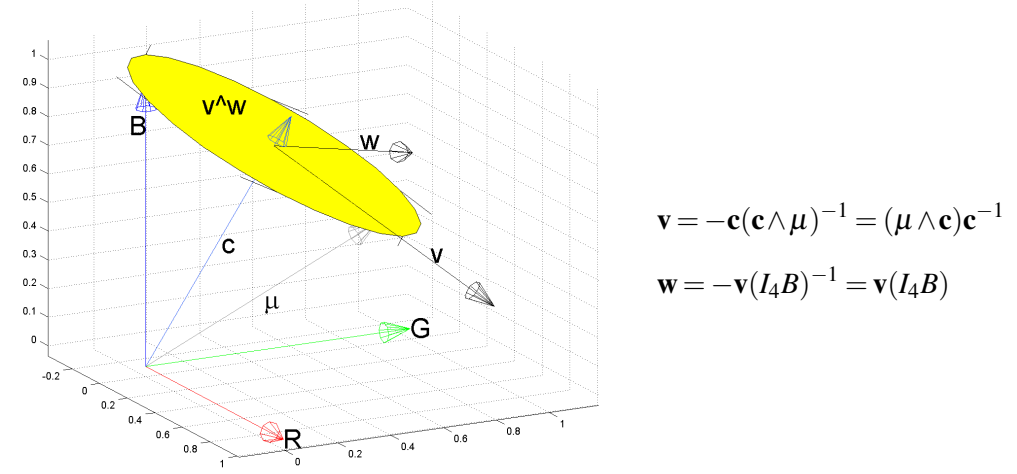

Fig. 1 Illustration of the basis $\left\{\mathbf{c}, \mathbf{e}_{\mathbf{4}}, \mathbf{v}, \mathbf{w}\right\}$ of $I_{4}$ using GABLE [4]

Depending on the intended application, it is not always necessary to reconstruct $\widehat{f_{B}}$ from $\widehat{f_{\| B}}$ and ${\widehat{f_{\perp B}}}^{2}$. If so, the following properties of the vectorial function $\widehat{f_{B}}$

$$
\begin{gathered}
\left(\widehat{\left.f_{\| B}(\mathbf{x})\right)_{B}}=\mathbf{c}\left[\left(\widehat{f_{B}}(\mathbf{x}) \cdot \mathbf{c}\right)+\left(\widehat{f_{B}}(\mathbf{x}) \cdot \mathbf{c} B\right) B\right]\right. \\
\left(\widehat{f_{\perp B}(\mathbf{x})}\right)_{B}=\mathbf{v}\left[\left(\widehat{f_{B}}(\mathbf{x}) \cdot \mathbf{v}\right)+\left(\widehat{f_{B}}(\mathbf{x}) \cdot \mathbf{v} I_{4} B\right) I_{4} B\right]
\end{gathered}
$$

give a set of four linear equations with four unknowns.

\section{Generalized Color Fourier Descriptors}

In this section, we propose an extension of the Generalized Fourier Descriptors of Smach et al. (initially dedicated to grayscale images) to color images.

\subsection{Generalized Fourier Descriptors (GFD)}

Generalized Fourier descriptors introduced by [15] are defined from the group action of $M_{2}$. This group is composed of translations and rotations on the plane. Two kinds of descriptors have been defined :

\footnotetext{
${ }^{2}$ More precisely, the two functions in the square brackets
} 
- "Spectral densities"-type invariants:

$$
I_{1}^{r}(f)=\int_{0}^{2 \pi}|\widehat{f}(r, \theta)|^{2} d \theta
$$

- "Shift of phases"-type invariants:

$$
I^{\xi_{1}, \xi_{2}}(f)=\int_{0}^{2 \pi} \widehat{f}\left(R_{\theta}\left(\xi_{1}+\xi_{2}\right)\right) \overline{\widehat{f}\left(R_{\theta}\left(\xi_{1}\right)\right) \widehat{f}\left(R_{\theta}\left(\xi_{2}\right)\right)} d \theta
$$

where $f$ is the image, $\widehat{f}(r, \theta)$ is the Fourier transform expressed in polar coordinates in the frequency plane, $\xi_{1}$ and $\xi_{2}$ are variables of the frequency plane and $R_{\theta}$ is a rotation of angle $\theta$. It must be emphasized that, by construction, $I_{1}^{r}$ and $I^{\xi_{1}, \xi_{2}}$ are strictly invariant in $\mathbb{R}^{2}$ with respect to the action of $M_{2}$.

Then, the descriptor vector for the first family of invariants, namely $I_{1}^{r}$, is defined as follows :

$$
G F D 1(f)=\left\{I_{1}^{0}(f)=|\hat{f}(0,0)|^{2}, \frac{I_{1}^{1}(f)}{I_{1}^{0}(f)}, \ldots, \frac{I_{1}^{m}(f)}{I_{1}^{0}(f)}\right\}
$$

where $m$ is the number of computed descriptors. In the same way, we define the $G F D 2$ descriptor vector from the second family of invariants $I^{\xi_{1}, \xi_{2}}$.

\subsection{Generalized Color Fourier Descriptors (GCFD)}

In order to deal with color images, a commonly used approach consists in computing descriptors on each color channel separately. Then, they are concatenated into a unique vector (e.g. [15]). This method implies three FFTs and three sets of descriptors. However, this marginal processing induces a loss of colorimetric information that can be avoided by using the color Clifford Fourier transform.

Equation (5) shows that the Clifford Fourier transform can be decomposed into two parts. So, two descriptor vectors are defined: $G C F D 1_{\| B}$ and $G C F D 1_{\perp B}$, each of them implying two complex FFT. According to the definition of $f_{\| B}$ :

$$
G C F D 1_{\| B}(f)=\left\{I_{\| B}^{0}(f)=\left|\hat{f}_{\| B}(0,0)\right|^{2}, \frac{I_{\| B}^{1}(f)}{I_{\| B}^{0}(f)}, \ldots, \frac{I_{\| B}^{m}(f)}{I_{\| B}^{0}(f)}\right\}
$$

where $I_{\| B}^{r}(f)=\int_{0}^{2 \pi}\left|\widehat{f_{\| B}}(r, \theta)\right|^{2} d \theta$ and $m$ is the number of computed descriptors. Similarly, $G C F D 1_{\perp B}$ is defined thanks to $\widehat{f_{\perp B}}$. Finally, the descriptor vector length is $2 \times m$ :

$$
G C F D 1_{B}(f)=\left\{G C F D 1_{\| B}(f), G C F D 1_{\perp B}(f)\right\}
$$

The same construction based on $I^{\xi_{1}, \xi_{2}}$ leads to $G C F D 2_{B}$. 


\section{Color Phase Correlation}

In the literature, the phase correlation [14] is a well-established method that is used for a lot of applications such as image recognition, video stabilization, motion estimation, stereo disparity analysis, vector flow analysis [5]. As it is based a direct application on the Fourier shift theorem, its definition depends on the chosen Fourier transform. Before presenting what can be a phase correlation method for color images, we recall now the principle of this method for grayscale images.

\subsection{Phase Correlation for Grayscale Images}

Let $f$ and $g$ be two grayscale images which are spatial shifted version of each one another. According to the Fourier shift theorem,

$$
\hat{g}(\mathbf{u})=\hat{f}(\mathbf{u}) \mathrm{e}^{\mathrm{i}\langle\mathbf{u}, \boldsymbol{\Delta}\rangle}
$$

Ideally, it is possible to extract the phase shift between $\hat{f}$ and $\hat{g}$ through the computation of their cross-power spectrum

$$
\begin{aligned}
R(\mathbf{u}) & =\frac{\widehat{f}(\mathbf{u}) \overline{\widehat{g}(\mathbf{u})}}{\mid \widehat{f}(\mathbf{u}) \overline{\widehat{g}(\mathbf{u}) \mid}}=\frac{\widehat{f}(\mathbf{u}) \overline{\hat{f}(\mathbf{u})} \mathrm{e}^{-\mathrm{i}\langle\mathbf{u}, \boldsymbol{\Delta}\rangle}}{\mid \widehat{f}(\mathbf{u}) \widehat{\widehat{f}(\mathbf{u})} \mathrm{e}^{-\mathrm{i}\langle\mathbf{u}, \boldsymbol{\Delta}\rangle \mid}} \\
& =\frac{|\widehat{f}(\mathbf{u})|^{2} \mathrm{e}^{-\mathrm{i}\langle\mathbf{u}, \boldsymbol{\Delta}\rangle}}{\left.|| \widehat{f}(\mathbf{u})\right|^{2} \mathrm{e}^{-\mathrm{i}\langle\mathbf{u}, \boldsymbol{\Delta}\rangle \mid}}=\frac{\mathrm{e}^{-\mathrm{i}\langle\mathbf{u}, \boldsymbol{\Delta}\rangle}}{\mid \mathrm{e}^{-\mathrm{i}\langle\mathbf{u}, \boldsymbol{\Delta}\rangle \mid}}=\mathrm{e}^{-\mathrm{i}\langle\mathbf{u}, \boldsymbol{\Delta}\rangle}
\end{aligned}
$$

where operator ${ }^{-}$is the usual complex conjugate. Ideally again, the exact translation $\boldsymbol{\Delta}=\left(\Delta_{1}, \Delta_{2}\right)$ can be obtained by taking the inverse Fourier transform of $R(\mathbf{u})$

$$
r(\mathbf{x})=\check{R}(\mathbf{u})=\delta_{-\Delta}
$$

where $\delta$ is the Dirac distribution.

In practice, the best estimated translation and correlation score $\rho$ are given by

$$
\boldsymbol{\Delta}=-\underset{\mathbf{x}}{\operatorname{argmax}}(|r(\mathbf{x})|) \quad \rho=\max _{\mathbf{x}}(|r(\mathbf{x})|)
$$

Coefficient $\rho$ should be equal to 1 when $g$ is a translated version of $f$ and it could be used as a similarity index between images in a recognition process. Note that the phase correlation method is invariant under translations but not under rotations. The invariance under rotations can be achieved by converting images in log-polar domain, but it will not be discussed here. 


\subsection{Phase Correlation for Color Images}

Phase correlation for color images is much more difficult than for grayscale images. A first tentative approach is to work directly on the relation between $\widehat{f}_{B}$ and $\widehat{g_{B}}$. According to (3) and for any unit bivector $B, \widehat{g_{B}}$ is ideally obtain by an isoclinic rotation of $\widehat{f}_{B}$. After some calculations similar to those of (14), it should be possible to obtained this rotation as a spinor represented by a multivector containing non vectorial terms. Unfortunately, definitions of the Fourier transform and Fourier inverse transform are not yet available for general multivectorial functions. So, an easier approach is to use the decompositions of $\widehat{f_{B}}$ and $\widehat{g_{B}}$ with respect to $B$.

According to (8) and (9), the phase correlation now relies on the detection of simultaneous Dirac at the same location:

$$
\begin{gathered}
R_{\| B}(\mathbf{u})=\frac{\widehat{f_{\| B}}(\mathbf{u}) \widehat{\widehat{g_{\| B}}(\mathbf{u})}}{\left|\widehat{f_{\| B}}(\mathbf{u}) \overline{\widehat{g_{\| B}}(\mathbf{u})}\right|}=\mathrm{e}^{-\langle\mathbf{u}, \boldsymbol{\Delta}\rangle B} \Longrightarrow r_{\| B}(\mathbf{x})=\delta_{-\boldsymbol{\Delta}} \\
R_{\perp B}(\mathbf{u})=\frac{\widehat{f_{\perp B}}(\mathbf{u}) \widehat{\widehat{g_{\perp B}}(\mathbf{u})}}{\mid \widehat{f_{\perp B}}(\mathbf{u}) \widehat{\widehat{g_{\perp B}}(\mathbf{u}) \mid}}=\mathrm{e}^{-\langle\mathbf{u}, \boldsymbol{\Delta}\rangle I_{4} B} \Longrightarrow r_{\perp B}(\mathbf{x})=\delta_{-\boldsymbol{\Delta}}
\end{gathered}
$$

In practice, one has to cope with the aggregation of $r_{\| B}$ and $r_{\perp B}$. Experiments section gives some results for different aggregation criteria. Whole process is illustrated in Fig. 2.

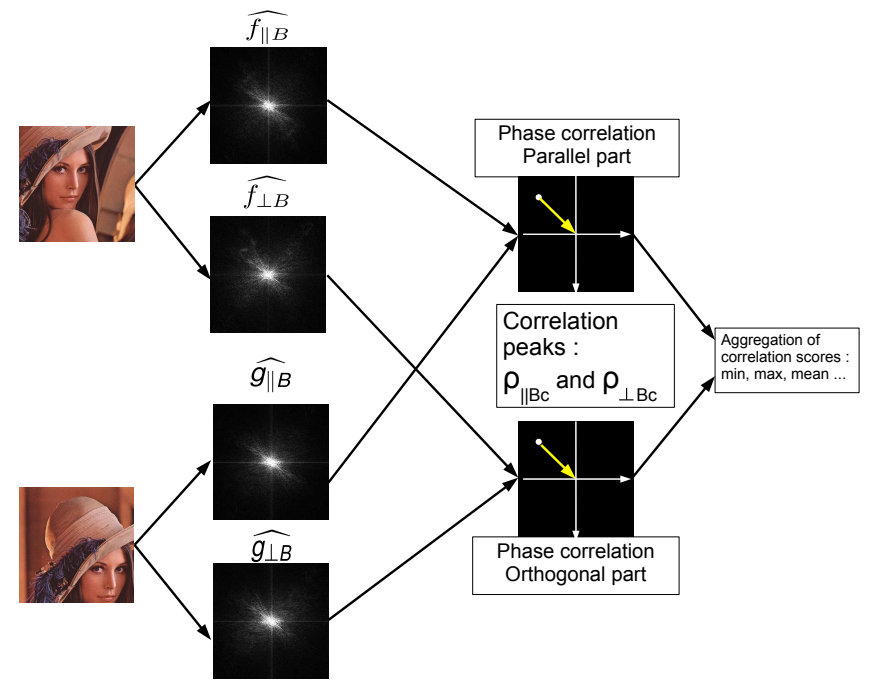

Fig. 2 Image similarity as a score aggregation

There are many rotations in $\mathbb{R}^{4}$ which map $\widehat{f_{B}}(\mathbf{u})$ to $\widehat{g_{B}}(\mathbf{u})$. Among these, one can choose the unique one that leaves invariant the plane generated by $\widehat{f}_{B}(\mathbf{u})$ and 
$\widehat{g_{B}}(\mathbf{u})$. This leads to the spinor $\tau(\mathbf{u})$ such that $\widetilde{g_{B}}(\mathbf{u})=\tau(\mathbf{u}) \widetilde{f}_{B}(\mathbf{u}) \tau^{-1}(\mathbf{u})$

$$
\tau_{B}(\mathbf{u})=\exp \left[\frac{\theta(\mathbf{u})}{2} \frac{\widetilde{g_{B}}(\mathbf{u}) \wedge \widetilde{f}_{B}(\mathbf{u})}{\left|\widetilde{g_{B}}(\mathbf{u}) \wedge \widetilde{f}_{B}(\mathbf{u})\right|}\right]=\frac{1+\widetilde{g_{B}}(\mathbf{u}) \widetilde{f}_{B}(\mathbf{u})}{\sqrt{2\left(1+\widetilde{g_{B}}(\mathbf{u}) \cdot \widetilde{f_{B}}(\mathbf{u})\right)}}
$$

where $\widetilde{f_{B}}(\mathbf{u})=\widehat{f_{B}}(\mathbf{u}) /\left|\widehat{f_{B}}(\mathbf{u})\right|$ and $\widetilde{g_{B}}(\mathbf{u})=\widehat{g_{B}}(\mathbf{u}) /\left|\widehat{g_{B}}(\mathbf{u})\right|$. This rotation is classified as a simple rotation by Lounesto [10]. Such an approach deviates from the conditions of the shift theorem by relaxing the constraint on the type of rotation between vectors $\widehat{f_{B}}$ and $\widehat{g_{B}}$. Here again, the inverse Fourier transform of $\tau(\mathbf{u})$ is not accessible. However, it is possible to neglect the bivectorial part of the spinor by transforming it to a constant bivector identifiable with complex imaginary i. A correlation score $\rho_{B}$ then can be built on $\theta(\mathbf{u})$ :

$$
R_{B}(\mathbf{u})=\mathrm{e}^{\mathrm{i} \theta(\mathbf{u})} \Longrightarrow \rho_{B}=\max _{\mathbf{x}}\left(\left|r_{B}(\mathbf{x})\right|\right)
$$

where $r_{B}(\mathbf{x})=\breve{R}_{B}(\mathbf{u})$. An alternative formulation of such a criterion is given by the cosine between $\widehat{f_{B}}$ and $\widehat{g_{B}}$ :

$$
\Re\left(R_{B}(\mathbf{u})\right)=\cos \left(\widehat{f_{B}}(\mathbf{u}), \widehat{g_{B}}(\mathbf{u})\right)=\frac{\widehat{f_{B}}(\mathbf{u}) * \widetilde{g_{B}}(\mathbf{u})}{\left|\widehat{f_{B}}(\mathbf{u})\right|\left|\widehat{g_{B}}(\mathbf{u})\right|}
$$

where $\sim$ and $*$ denote the reverse operator and the Hestenes scalar product [8].

\section{Experimentations}

Our goal is to evaluate if proposed descriptors are good at classifying images. More precisely, the unique label of the request image is predicted from an entire set of labeled images. Both synthetic and standard image databases are considered and the choice of the bivector is discussed.

\subsection{Image database}

The databases used in this section are COIL-100, color FERET and, to check robustness again noise, a noisy version of this last dataset.

- COIL-100 (Columbia Object Image Library) database [12] is composed of 7200 color images of size $128 \times 128$ of 100 different objects. Each picture has been taken with a black background and 72 different angles of view. This database, used in similar works [15], can be qualified as "easy" from an image classification context as every image background is removed. 
- color FERET database [13] is composed of face images of 1408 different persons, taken from different angles of view. In our tests, a set of 2992 images containing 272 persons equally represented by 11 pictures is selected and size of images is reduced to $128 \times 128$. This database is more difficult than the first one due to background illumination changes.

- noisy color FERET database is derived from the color FERET database, but Gaussian noise is added to each color plane of the images. The parameter $\theta$ is fixed to 0.23 , which is the maximum noise level used in [15].

\subsection{Descriptors Extraction}

Regarding the $G F D, 64$ descriptors are extracted for each color channel. For $G F D 1$, it consists of 64 values of radius $r$ in $I_{1}^{r}$ and GFD2 is built from equally spaced values of $\xi_{2}$ in its polar domain $[0,2 \pi] \times[1,8]$ and $\xi_{1}$ set to $(0,1)$. As it is argued in [15], such $\xi_{1}$ and $\xi_{2}$ choices allow to take into account low frequencies of the image, i.e. the shape. For GCFD1 and GCFD2, the length of the descriptor vectors are $64 \times 2$ (parallel and orthogonal part of the Clifford Fourier transform). Regarding the phase correlation, one score corresponding to the correlation peak is extracted for each image pair. Two cases are considered: either phase correlation is computed from parallel and orthogonal parts of the Clifford Fourier transform, and the two correlation scores, $\rho_{\| B}$ and $\rho_{\perp B}$, are aggregated to obtain a single score, either the correlation $\rho_{B}$ is computed from reconstructed Fourier transform.

\subsection{Classification}

The classification step is performed using a standard SVM (Support Vector Machine) [2]. As GFD and GCFD are vector descriptors they can directly be used as input for such a kernel based classifier. In this paper a RBF kernel is selected and the value of the two parameters $\theta$ and $C$ are empirically determined to maximize the recognition rates for each database. The phase correlation methods directly result in a real valued score assessing the matching quality and this similarity measure can not be used as input in a standard SVM. Various solutions to address such cases have been proposed in the literature (see [3] for a recent review). Fortunately, in the phase correlation case, one can slightly modify the SVM algorithm by replacing the inner product values of the Gram matrix by a symmetric similarity measure ensuring its semi-positive definiteness. Such a property is guaranteed by taking as a measure the mean value of the correlation scores between $\hat{f}_{B}$ and $\hat{g}_{B}$ and between $\hat{g}_{B}$ and $\hat{f}_{B}$. Validation of the classification results is done by a 10 -fold cross-validation. 


\subsection{Evaluation of the GCFD}

In this section, the Generalized Clifford based Fourier Descriptors, GCFD1 and $G C F D 2$, are evaluated to assess their classification performance relatively to the usual Generalized Fourier Descriptors, GFD1 and GFD2. The classification performance of GFD and GCFD is tested on the COIL-100, the color FERET and the noisy color FERET database. Each database (see Table 1, Table 2, Table 3) is processed using the same choices of bivectors, one bivector per row of the Tables. The first one, denoted by $B_{r}$, is obtained using a red vector (i.e. $B_{r}=\mathbf{r} \wedge \mathbf{e}_{4}$ ), the three next bivectors $B_{g}, B_{b}, B_{\mu}$ respectively refer to green, blue colors and the achromatic axis. The next row gives the best classification rate, the mean and standard deviation of classification rates obtained by randomly choosing 100 bivectors. These five first rows provide results for single bivectors while the last two concern triple size bivectors. The first one, denoted by $B_{r}+B_{g}+B_{b}$, uses as descriptor the concatenation of descriptors computed from $B_{r}, B_{g}$ and $B_{b}$ (descriptors of size 384). The last row, denoted by $B_{1}+B_{2}+B_{3}$, provides results obtained by using an automatic selection algorithm to select the three bivectors maximizing the classification rate. This socalled "SFFS" (Sequential Floating Forward Selection) algorithm is a suboptimal selection procedure that avoid exhaustive search in the feature space, here defined by the space of the $c$ normalized color vectors. Interested reader can refer to [9], for comparison to similar optimization techniques.

COIL-100 being an easy dataset from a classification point of view, any descriptors provides excellent results, very close to $100 \%$. In Table 1, the descriptors size is recalled for each method and best results for each method is highlighted. Yellow color highlights best results for one bivector and green highlights results for 3 bivectors (obtained by concatenation or by the SFFS procedure). It must be noted that such a classification rate validates the choice of Fourier descriptors on this kind of images where color background is homogeneous and similar for every image. The standard deviations obtained for $B_{\text {rand }}$ are small and suggest little influence of the bivector choice on this database and experiments were not carried further in that direction.

\begin{tabular}{|l||c|c|c|c|}
\hline \multicolumn{1}{|c||}{} & \multicolumn{4}{c|}{ COIL-100 } \\
\hline Bivectors & GFD1(64 desc.) & GCFD1(128 desc.) & GFD2(64 desc.) & GCFD2(128 desc.) \\
\hline$B_{r}$ & 98.04 & 99.83 & 98.69 & 99.81 \\
\hline$B_{g}$ & 98.06 & 99.56 & 99.39 & 99.85 \\
\hline$B_{b}$ & 96.90 & 99.86 & 94.03 & 99.85 \\
\hline$B_{\mu}$ & 98.49 & 99.25 & 99.40 & 99.47 \\
\hline$B_{\text {rand }}(\times 100)$ & $98.42 \pm 0.3$ & $99.54 \pm 0.3$ & $98.88 \pm 0.82$ & $99.82 \pm 0.1$ \\
\multicolumn{1}{|c||}{ max. } & 98.87 & 99.89 & 99.47 & 99.96 \\
\hline \hline & 192 desc. & 384 desc. & 192 desc. & 384 desc. \\
\hline$B_{r}+B_{g}+B_{b}$ & 99.9 & 99.92 & 99.89 & 99.87 \\
\hline$B_{1}+B_{2}+B_{3}$ (SFFS) & 99.86 & 99.96 & 99.89 & 99.96 \\
\hline
\end{tabular}

Table 1 COIL-100 : Recognition rates in \% with GFD1, GCFD1, GFD2 and GCFD2. 


\begin{tabular}{|l||c|c|c|c|}
\hline \multicolumn{1}{|c||}{} & \multicolumn{4}{c|}{ color FERET } \\
\hline Bivectors & GFD1(64 desc.) & GCFD1(128 desc. $)$ & GFD2(64 desc.) & GCFD2(128 desc.) \\
\hline$B_{r}$ & 76.70 & 87.90 & 77.31 & 84.42 \\
\hline$B_{g}$ & 73.66 & 79.65 & 77.37 & 80.01 \\
\hline$B_{b}$ & 70.49 & 84.49 & 75.87 & 82.31 \\
\hline$B_{\mu}$ & 73.03 & 78.10 & 77.30 & 82.12 \\
\hline$\left.B_{\text {rand }} \times 100\right)$ & $73.72 \pm 1$ & $85.34 \pm 2.92$ & $77.54 \pm 0.69$ & $84.50 \pm 2.06$ \\
\multicolumn{1}{|c||}{$\operatorname{max.}$} & 76.14 & 90.37 & 78.91 & 89.57 \\
\hline \hline & 192 desc. & 384 desc. & 192 desc. & 384 desc. \\
\hline$B_{r}+B_{g}+B_{b}$ & 88.03 & 85.53 & 84.26 & 82.55 \\
\hline$B_{1}+B_{2}+B_{3}$ (SFFS) & 85.46 & 93.15 & 82.89 & 90.07 \\
\hline
\end{tabular}

Table 2 Color FERET : Recognition rates in \% with GFD1, GCFD1, GFD2 and GCFD2

Regarding the color FERET dataset, it can be checked that the GCFD1 outperforms both $G F D 1$ and $G F D 2$ in term of classification rate for any bivector choice. $G C F D 2$ while providing better results than GFD1 and GFD2 is not better than $G C F D 1$. This may be due to the choice of the $\xi_{1}$ and $\xi_{2}$ parameters, but none of our experiments led to an improvement in that respect. Anyway, these results clearly show the added benefit of the Clifford Fourier transform with regard to classification. Concatenation $\left(B_{r}+B_{g}+B_{b}\right)$ of bivectors improves the results for $G F D$ but not for $G C F D$. This is probably due to the better information encoding done by the Clifford Fourier transform and GCFD descriptors obtained for various bivectors are probably more redundant than the marginal GFD. This is confirmed by the SFFS method: selection of 3 "optimal" bivectors pushes $G C F D$ to the best results. In the random bivector choice experiment, GCFD1 and GCFD2 standard deviation are quite important compared to the ones of $G F D 1$ and $G F D 2$. This clearly reveals that the GCFD depends on the choice of the bivector. To inspect its influence, the random experiment results are detailed in Fig. 3. Color of each dot denotes the $c$ color chosen to define the bivector, and best ones are mostly blue. Visual inspection of the database confirms that this color corresponds to the background color of most images. As the bivector is unique for a given database it must be chosen either according to some prior knowledge either according to an empirical search method like SFFS.

noisy color FERET: Influence of noise on classification rate is given in Table 3. $G F D 1$ is the most sensitive to noise and, as expected (see [15]), GFD2 is much more robust.

\begin{tabular}{|c|c|c|c|c|}
\hline & \multicolumn{4}{|c|}{ noisy color FERET } \\
\hline Bivectors & GFD1(64 desc.) & $G C F D 1(128$ desc. $)$ & GFD2(64 desc.) & $G C F D 2(128$ desc. $)$ \\
\hline$B_{r}$ & 45.32 & 71.05 & 73.46 & 83.49 \\
\hline$B_{g}$ & 46.83 & 61.99 & 75.26 & 78.64 \\
\hline$B_{b}$ & 48.49 & 73.46 & 74.77 & 81.78 \\
\hline$B_{\mu}$ & 55.28 & 62.03 & 77.34 & 80.98 \\
\hline$B_{\text {rand }}(\times 100)$ & $54.23 \pm 1.75$ & $69.64 \pm 3.21$ & $76.59 \pm 0.74$ & $82.56 \pm 1.80$ \\
\hline $\max$. & 57.55 & 77.27 & 78.41 & 87.00 \\
\hline & 192 desc. & 384 desc. & 192 desc. & 384 desc. \\
\hline$B_{r}+B_{g}+B_{b}$ & 73.16 & 72.16 & 83.25 & 81.12 \\
\hline$B_{1}+B_{2}+B_{3}$ (SFFS) & 71.52 & 80.62 & 83.36 & 88.24 \\
\hline
\end{tabular}

Table 3 noisy Color FERET : Recognition rates in \% with GFD1, GCFD1, GFD2 and GCFD2 


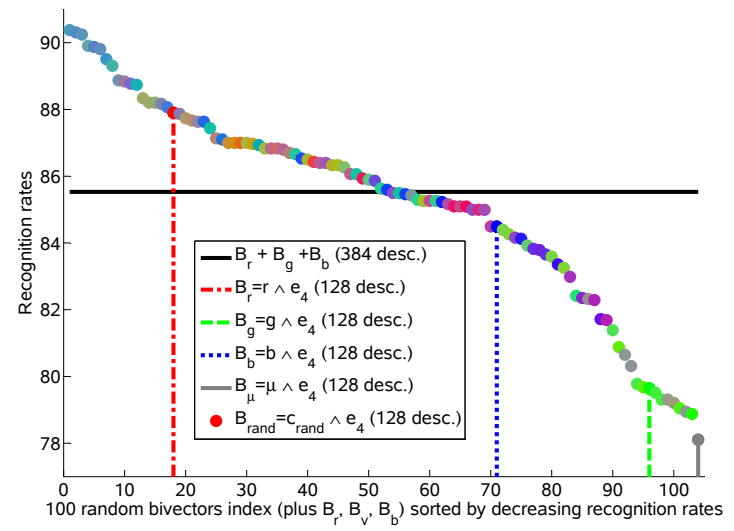

Fig. 3 color FERET: Recognition rates with GCFD1 for 100 random bivectors

\subsection{Evaluation of the phase correlation}

In this paper, two methods have been proposed to compute phase correlation for color images, both depend on a bivector $B_{c}$, where $c$ is the chosen color. The first one depends on two correlation scores, $\rho_{\| B_{c}}$ and $\rho_{\perp B_{c}}$, given by the parallel and orthogonal part of the Clifford Fourier transform and requires an aggregation step to give final score. The second one, noted $\rho_{B_{c}}$, does not require such a processing.

Synthetic data: Two simple images (see Fig. 4) are considered, they contain the same shape (a rectangle) on a black background, but the second is translated and its color $\mathbf{c}_{2}$ changed from experiments to experiments. Bivectors $B_{\mu}, B_{r}$ and $B_{c_{1}}$, where $\mathbf{c}_{\mathbf{1}}$ is the color of the first rectangle, are used to compute the correlation scores. In

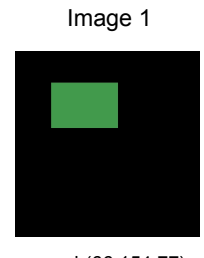

$c_{1}=\operatorname{rgb}(66,154,77)$

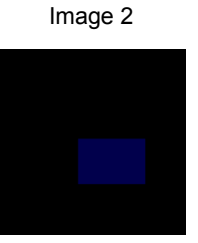

$c_{2}$

Fig. 4 Synthetic data: rectangles of color $\mathbf{c}_{\mathbf{1}}$ and $\mathbf{c}_{\mathbf{2}}$

Table 4, the '-' symbol denotes a value that cannot be computed because the parallel (resp. orthogonal) part is null. Taking the example in which $c_{2}=\operatorname{rgb}(66,0,0)$ one can see that $\rho_{\| B_{c_{2}}}=\rho_{\perp B_{c_{2}}}=1$. This is not conclusive as two rectangles which have different colors must be considered as different. However, the correlation score $\rho_{B_{c_{2}}}$ computed from the reconstructed Clifford Fourier transform gives scores lower than one and depends on the amount of color that the two rectangles have in common. 


\begin{tabular}{|c|c|c|c|c|c|c|c|c|c|c|c|c|c|c|c|}
\hline \multirow{2}{*}{$\mathbf{c}_{\mathbf{c}_{2}}{ }^{B}$} & \multicolumn{3}{|c|}{$B_{\mu}$} & \multicolumn{3}{|c|}{$B_{r}$} & \multicolumn{3}{|c|}{$B_{g}$} & \multicolumn{3}{|c|}{$B_{b}$} & \multicolumn{3}{|c|}{$B_{c_{1}}$} \\
\hline & $\rho_{\| B}$ & $\rho_{\perp B}$ & $\rho_{B}$ & $\rho_{\| B}$ & $\rho_{\perp B}$ & $\rho_{B}$ & $\rho_{\| B}$ & $\rho_{\perp B}$ & $\rho_{B}$ & $\rho_{\| B}$ & $\rho_{\perp B}$ & $\rho_{B}$ & $\rho_{\| B}$ & $\rho_{\perp B}$ & $\rho_{B}$ \\
\hline$c_{1}$ & 1 & 1 & 1 & 1 & 1 & 1 & 1 & 1 & 1 & 1 & 1 & 1 & 1 & - & 1 \\
\hline $\operatorname{rgb}(66,0,0)$ & 1 & 1 & 0.43 & 1 & - & 0.36 & - & 1 & 0.55 & - & 1 & 0.91 & 1 & - & 0.36 \\
\hline$r g b(0,154,0)$ & 1 & 1 & 0.84 & - & 1 & 0.93 & 1 & - & 0.83 & - & 1 & 0.91 & 1 & - & 0.84 \\
\hline$r g b(0,0,77)$ & 1 & 1 & 0.50 & - & 1 & 0.93 & - & 1 & 0.55 & 1 & - & 0.42 & 1 & - & 0.42 \\
\hline$\mu$ & 1 & - & 0.93 & 1 & 1 & 0.96 & 1 & 1 & 0.93 & 1 & 1 & 0.97 & 1 & - & 0.93 \\
\hline
\end{tabular}

Table 4 Correlation scores between image 1 and 2 for various choices of $\mathbf{c}_{2}$ and $B$. From image 1 to 2 , the rectangle has color changed from $\mathbf{c}_{\mathbf{1}}=\operatorname{rgb}(66,154,77)$ to $\mathbf{c}_{\mathbf{2}}$ and a translation is applied.

The same remarks apply to $c_{2}$ taken equal to $\operatorname{rgb}(0,154,0)$ or $\operatorname{rgb}(0,0,77)$. The behavior for the gray axis level, $\mu$, is different as $\rho_{B_{\mu}}$ is always high: unsurprisingly, it mostly depends on the shape without taking into account color information.

\begin{tabular}{|c|c||c|c|c|c|c||c|}
\hline$\rho$ & $\rho_{\| B}$ & $\rho_{\perp B}$ & $\rho_{\|, \perp B}^{\min }$ & $\rho_{\|, \perp B}^{\max }$ & $\rho_{\|, \perp B}^{\text {mean }}$ & $\rho_{B}$ \\
\hline 94.96 & $B_{r}$ & 95.38 & 96.33 & 96.46 & 96.14 & 96.58 & 97.50 \\
& $B_{g}$ & 95.29 & 96.79 & 96.68 & 95.90 & 96.68 & 97.49 \\
$B_{b}$ & 95.08 & 96.58 & 96.60 & 95.89 & 96.51 & 97.49 \\
$B_{c_{i}}$ & 95.33 & 83.92 & 82.60 & 96.08 & 95.50 & 97.53 \\
\hline
\end{tabular}

Table 5 COIL-100 : Recognition rates in $\%$ with the phase correlation for color images

COIL-100: Results with $B_{r}, B_{g}, B_{b}$ and $B_{c_{i}}$ bivectors are given. Notice that $c_{i}$ corresponds to the choice of one bivector per request image, this color being the dominant color of the image. Table 5 clearly shows that the correlation computed from the reconstructed Clifford Fourier transform is the best method for this database. It has more discriminative power for color objects than other methods, and more importantly, it seems to be quite insensitive to the bivector choice. One can also see that most of color phase correlation methods give better recognition rates than the classical phase correlation for grayscale images. The different choices of bivectors or aggregation functions do not give really improve the results nevertheless recognition rates are high.

\begin{tabular}{|c|c||c|c|c|c|c||c|}
\hline$\rho$ & $\rho_{\| B}$ & $\rho_{\perp B}$ & $\rho_{\|, \perp B}^{\min }$ & $\rho_{\|, \perp B}^{\max }$ & $\rho_{\|, \perp B}^{\text {mean }}$ & $\rho_{B}$ \\
\hline & 66.37 \\
\hline$B_{r}$ & 66.51 & 66.00 & 67.64 & 68.05 & 69.15 & 66.74 \\
$B_{g}$ & 66.00 & 67.91 & 67.31 & 66.95 & 67.51 & 66.41 \\
$B_{b}$ & 65.57 & 67.41 & 68.01 & 66.95 & 67.71 & 66.81 \\
$B_{c_{i}}$ & 66.34 & 74.50 & 76.84 & 71.52 & 78.38 & 66.94 \\
\hline
\end{tabular}

Table 6 color FERET : Recognition rates in \% with the phase correlation for color images

One can see on the Table 6 that the results for the color FERET database are quite different. Indeed, $\rho_{B_{r}}$ is not the best method anymore, but still very stable. This relatively low performance is due to confusions induced by the different colors constituting the background. So, if the reconstructed Clifford Fourier transform is considered, all the color information is aggregated. Hence the choice of one bivector per request image, corresponding to the dominant color, separates the background and the foreground. This choice is the best among $B_{r}, B_{g}$ and $B_{b}$ and gives the best recognition rate using the mean as an aggregation function. 


\section{Conclusion}

Two descriptors for color object recognition based on Clifford Fourier transform and with the viewpoint of group actions are proposed. Better classification results than those of analogous marginal methods are provided. Specially, Clifford Fourier descriptors enhance Generalized Fourier Descriptors with lower computation cost and size (only two FFT instead of three). Mathematically sound phase correlation computation for color images would imply an inverse Clifford Fourier transform of a spinor which is not available for now. Although some workaround are proposed in this paper, future works will give more efficient and consistent solutions.

Acknowledgements This work is partially supported by the ONR Grant N00014-09-1-0493 and "La Région Poitou-Charentes".

\section{References}

1. Batard, T., Berthier, M., Saint-Jean, C.: Clifford fourier transform for color image processing. In: E. Bayro-Corrochano, G. Scheuermann (eds.) Geometric Algebra Computing in Engineering and Computer Science, chap. 8, pp. 135-161. Springer Verlag (2010)

2. Chang, C.C., Lin, C.J.: Libsvm: a library for support vector machines (2001). Software available at http://www.csie.ntu.edu.tw/ cjlin/libsvm

3. Chen, Y., Garcia, E.K., Gupta, M.R., Rahimi, A., Cazzanti, L.: Similarity-based classification: Concepts and algorithms. Journal of Machine Learning Research 10, 747-776 (2009)

4. Dorst, L., Mann, S., Bouma, T.: Gable: A matlab tutorial for geometric algebra (2002)

5. Ebling, J., Scheuermann, G.: Clifford fourier transform on vector fields. IEEE Transactions on Visualization and Computer Graphics 11, 469-479 (2005)

6. Ell, T.A., Sangwine, S.J.: Hypercomplex fourier transforms of color images. IEEE Transactions on Image Processing 16(1), 22-35 (2007)

7. Flusser, J., Suk, T., Zitova, B.: Moments and Moment Invariants in Pattern Recognition. Wiley (2009)

8. Hestenes, D., Sobczyk, G.: Clifford Algebra to Geometric Calculus. Reidel (1984)

9. Jain, A., Zongker, D.: Feature-selection: Evaluation, application, and small sample performance. IEEE Trans. on Pattern Analysis and Machine Intelligence 19(2), 153-158 (1997)

10. Lounesto, P.: Clifford Algebras and Spinors, 2nd edn. Cambridge University Press (2001)

11. Lowe, D.G.: Object recognition from local scale-invariant features. In: International Conference on Computer Vision 99, pp. 1150-1157 (1999)

12. Nene, S.A., Nayar, S.K., Murase, H.: Columbia object image library (coil-100) (1996). Technical Report CUCS-006-96

13. Phillips, P.J., Wechsler, H., Huang, J., Rauss, P.: The feret database and evaluation procedure for face recognition algorithms. Image and Vision Computing 16(5), 295-306 (1998)

14. Reddy, B., Chatterji, B.: An fft-based technique for translation, rotation, and scale-invariant image registration. IEEE Transactions on Image Processing 5(8), 1266 -1271 (1996)

15. Smach, F., Lemaître, C., Gauthier, J.P., Miteran, J., Atri, M.: Generalized fourier descriptors with applications to objects recognition in svm context. Journal of Mathematical Imaging and Vision 30(1), 43-71 (2008) 